\title{
Bioinformatic prediction of the epitopes of Echinococcus granulosus antigen 5
}

\author{
WEI PAN $^{1^{*}}$, DE-SHENG CHEN ${ }^{1,2^{*}}$, YUN-JUAN LU $^{1,2}$, FEN-FEN SUN $^{1}$, HUI-WEN XU ${ }^{1,2}$, \\ YA-WEN ZHANG $^{1,2}$, CHAO YAN $^{1}$, LIN-LIN FU ${ }^{1}$, KUI-YANG ZHENG ${ }^{1}$ and REN-XIAN TANG ${ }^{1}$ \\ ${ }^{1}$ Department of Pathogenic Biology and Immunology, Laboratory of Infection and Immunity; \\ ${ }^{2}$ Department of Clinical Medicine, Xuzhou Medical University, Xuzhou, Jiangsu 221004, P.R. China
}

Received December 13, 2015; Accepted December 5, 2016

DOI: $10.3892 /$ br.2016.827

\begin{abstract}
The aim of the present study was to predict and analyze the secondary structure, and B and T cell epitopes of Echinococcus granulosus antigen 5 (Ag5) using online software in order to investigate its immunogenicity and preliminarily evaluate its potential as an effective antigen peptide vaccine for cystic echinococcosis. The PortParam program was used to analyze molecular weight, the theoretical isoelectric point, instability index and other physicochemical properties. The secondary structure of the $\mathrm{Ag} 5$ protein was predicted using Self-Optimized Prediction method With Alignment and the tertiary structure of the Ag5 protein was predicted using 3DLigandSite together with Center for Biological Sequence Analysis Prediction Servers. Furthermore, the Immune Epitope Database software was used to predict B cell epitopes, and $\mathrm{T}$ cell epitopes were predicted with the BioInformatics and Molecular Analysis Section and SYFPEITHI programs. The results demonstrated that $\alpha$-helixes, $\beta$-turns, random coils and extended strands account for 23.35, 10.95, 41.32, and $24.38 \%$ of the secondary structure of the $\mathrm{Ag} 5$ protein, respectively. Ten potential B cell epitopes of Ag5 were identified as the amino acids sequences 27-39, 70-80, 117-130, 146-168, 250-262, 284-293, 339-349, 359-371, 403-412 and 454-462, and seven potential $\mathrm{T}$ cell epitopes were identified as the amino acid sequences 52-60, 57-65, 182-190, 231-239, 273-281, 318-326 and 467-475. Thus, ten B cell epitopes and seven $\mathrm{T}$ cell epitopes were identified on Ag5, suggesting the
\end{abstract}

Correspondence to: Professor Kui-Yang Zheng or Professor Ren-Xian Tang, Department of Pathogenic Biology and Immunology, Laboratory of Infection and Immunity, Xuzhou Medical University, 209 Tongshan Road, Xuzhou, Jiangsu 221004, P.R. China

E-mail: zky02@163.com

E-mail: tangrenxian-t@163.com

*Contributed equally

Key words: Echinococcus granulosus, antigen 5, antigen epitope, bioinformatics strong immunogenicity of this protein, which could be applied to design antigen peptide vaccines for echinococcosis.

\section{Introduction}

Echinococcosis, also termed cystic echinococcosis, is a type of zoonosis caused by the larval stages of Echinococcus granulosus (E. granulosus). This disease is endemic and widespread throughout the world (1-4). In China, it is mainly endemic in large pastoral areas of northwestern regions, such as Xinjiang, Gansu, Qinghai, and Inner Mongolia, as well as in southwestern areas, such as Sichuan and Tibet (5). The life cycle of this cestode involves dogs, wolves and other canids as definitive hosts, and intermediate hosts are usually sheep, cattle, goats and pigs. However, humans may become accidental intermediate hosts when food and water sources are contaminated by the eggs of E. granulosus. Echinococcosis causes significant harm to human health and hampers the development of local animal husbandry. At present, many actions have been taken to cure this disease; however, few are sufficiently effective to cure it completely. Surgery in combination with chemotherapy has been identified as most efficacious and has become the first choice of therapy. However, this therapeutic strategy inevitably entails surgical risk and requires considerable labour, material and financial resources (6). An earlier diagnosis correlates to more effective surgery and chemotherapy. Therefore, early diagnosis is of great importance to the treatment of echinococcosis. However, early diagnosis continues to be an obstacle for doctors, as it is difficult to confirm the larvae using imaging diagnosis (7-10). Immunological methods are more promising, however, to the best of our knowledge, no studies regarding the early diagnosis of the E. granulosus infection have been performed. Therefore, there is an urgent requirement to develop effective preventative and treatment strategies.

A study by Lightowlers et al (11) revealed that the $\mathrm{Eg} 95$ recombinant antigen induced a $95-100 \%$ protective immune effect against oncospheres in sheep. Thus, immune prevention may be an effective measure that could be taken to prevent cystic echinococcosis. Strohmaier et al (12) identified the immunogenic amino acid sites of the foot and mouth disease virus and thereby designed novel epitope vaccines. With the rapid development of molecular biology and immunology, epitope vaccines have become the focus of research on 
molecular vaccines (13). To prevent infection by Echinococcus multilocularis (E. multilocularis), Kouguchi et al (14) developed the Emy162 recombinant antigen, which induced a $74.3 \%$ protective rate in rats against E. multilocularis. In addition, Katoh et al (15) developed a vaccine based on the Em95 protein, and the protective efficiency of the vaccine was $78.5-82.9 \%$. These results suggest that the prevention of echinococcosis using a molecular vaccine is feasible.

Antigen 5 (Ag5) is one of the dominant antigens of E. granulosus cyst fluids. It is a dimeric protein composed of 22- and 38-kDa subunits linked by a disulphide bridge, with the two subunits bearing an N-glycan modification $(16,17)$. The 38-kDa subunit is closely associated with serine proteases of the trypsin family (16). It has been confirmed that $\mathrm{Ag} 5$ is expressed in all stages of the life cycle of E. granulosus (18). Ag5 is strongly expressed in the protoscolex tegument, the embryonic membrane of eggs, and the surface of oncospheres; it is also expressed weakly in the adult tegument. As a result of this, the present study hypothesized that $\mathrm{Ag} 5$ may have diagnostic value and serve as a candidate antigen for a vaccine against cystic echinococcosis. To the best of our knowledge, recent studies regarding Ag5 have primarily focused on the diagnostic evaluation of cystic echinococcosis (16-19). Yarzabal et al (20) demonstrated the occurrence of cross-reactions in ELISA detection between Ag5 and other parasites, such as E. multilocularis and certain other helminthes. This result revealed the low specificity of this protein, thus $\mathrm{Ag} 5$ could not be widely applied in clinical diagnosis. However, whether Ag5 may be a candidate vaccine for cystic echinococcosis remains unclear.

In the current study, computer technology and online molecular biology software were used to predict various characteristics of Ag5, particularly the B and T cell epitopes, to analyze its immunogenicity and lay the theoretical foundation for further investigation of epitope vaccines against cystic echinococcosis.

\section{Materials and methods}

Amino acid sequence of Ag5. The nucleotide sequence of Ag5 was selected from GenBank (NIH, Bethesda, MD, USA; GenBank no. JF970202). The amino acid sequence of Ag5 was predicted by DNAman software (LynnonBiosoft, San Ramon, CA, USA).

Prediction of the Ag5 protein secondary structure. The secondary structure of $\mathrm{Ag} 5$ protein was predicted using the Self-Optimized Prediction method With Alignment (SOPMA) Server (https://npsa-prabi.ibcp.fr/cgibin/npsa_automat. pl?page=npsa_sopma.html) (21). The sequence of Ag5 protein was input, then four conformational states were predicted. The transmembrane structure of Ag5 protein was predicted by TMHMM Server v. 2.0 (http://www.cbs.dtu.dk/services/ $\mathrm{TMHMM} /$ ) (22). The sequence of $\mathrm{Ag} 5$ protein was input, and three regions, including inside, transmembrane and outside regions, were analyzed.

Prediction of the Ag5 protein tertiary structure. The tertiary structure of Ag5 protein was predicted using the online prediction server 3DLigandSite (http://www.sbg.bio.ic.ac. uk) (23). To improve the accuracy of the prediction, the tertiary
MARSRPLWIVFVCLFATAALGLELTLDPDELVKAQRESHGGFYFYDSN GATLMFNRSLFVYRENIYDGWSRWSECSPHTCLEHRYRRCVDDSYTQ PVNYLTSSARICPFKYIAEERPCEDKSNCIINSKPSEELTRMQEKCGIRGS FDKNTAKPSRRRWKDMDDDEADDAEAEERGEYESERLPYSLKILGGK SAKSKSWPWHVGIYKAANYNASEGLTRLKSENIICGGTLITPRWVLTA AHCLKPIFGSSNALPFGIPAPLNTDEMKPIFLLVRAGDTVLEGTRTRNEQ ESDHVVDLVVIHPDWVAQRVDSPFDVALLRLETPVNIESDGAGVACVP KNADATPAEDAVCFSVGWGEKSRPISKPRRRRPTFFNPFFWPFGRLWER RPQRPTSLNEIRVSIDPPEKCFHHDDENEAQICAGSSNKGVCAGDTGGG LFCRNEEDGRWYVYGVMGSGPTQYCKSRRWLYNSVGSVIQWINRYAV

Figure 1. Amino acid sequence encoded by the Ag5 gene.

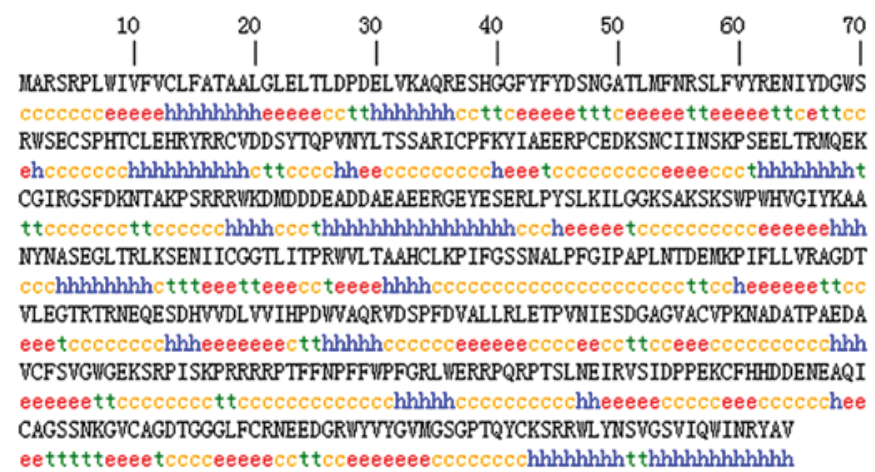

Sequence length : 484

SOPMA :

$\begin{array}{lcr}\text { Alpha helix } & (\mathrm{Hh}): & 113 \text { is } 23.35 \% \\ 3_{10} \text { helix } & (\mathrm{Gg}): & 0 \text { is } 0.00 \% \\ \mathrm{Pi} \text { helix } & (\mathrm{Ii}): & 0 \text { is } 0.00 \% \\ \text { Beta bridge } & (\mathrm{Bb}): & 0 \text { is } 0.00 \% \\ \text { Extended strand } & (\mathrm{Ee}): & 118 \text { is } 24.38 \% \\ \text { Beta turn } & (\mathrm{Tt}): & 53 \text { is } 10.95 \% \\ \text { Bend region } & (\mathrm{Ss}): & 0 \text { is } 0.00 \% \\ \text { Random coil } & (\mathrm{Cc}): & 200 \text { is } 41.32 \% \\ \text { Anbiguous states }(?): & 0 \text { is } 0.00 \% \\ \text { Other states } & : & 0 \text { is } 0.00 \%\end{array}$

Figure 2. Secondary structure of Ag5 using SOPMA software. SOPMA, Self-Optimized Prediction method With Alignment.

structure was also predicted using an additional online software application, Center for Biological Sequence Analysis (CBS) Prediction Servers (http://www.cbs.dtu.dk/services) (24). The 3DLigandSite software used homology modelling to predict the tertiary structure, as did the CBS Prediction Servers. When the sequence of Ag5 was input, a high homology model was output.

\section{Prediction of Ag5 protein epitopes}

Prediction of the Ag5 protein B cell epitopes. The B cell epitopes of the Ag5 protein were predicted using the online prediction software Immune Epitope Database (IEDB; http://tools.immuneepitope.org/bcell/) (25). The sequence of Ag5 protein was input, and then the thresholds were all set to 1.0. The other parameters were not changed.

Prediction of the Ag5 protein $T$ cell epitopes. Major histocompatibility complex (MHC)-I human leukocyte antigen (HLA)-A*0201-restricted T cell epitopes were predicted by the online prediction software BioInformatics and Molecular Analysis Section (BIMAS; http://www-bimas.cit.nih.gov/molbio/hla_bind/) (26) together with SYFPEITHI (http://www.syfpeithi.de/bin/MHCServer. 
TMHMM posterior probabilities for WEBSEQUENCE

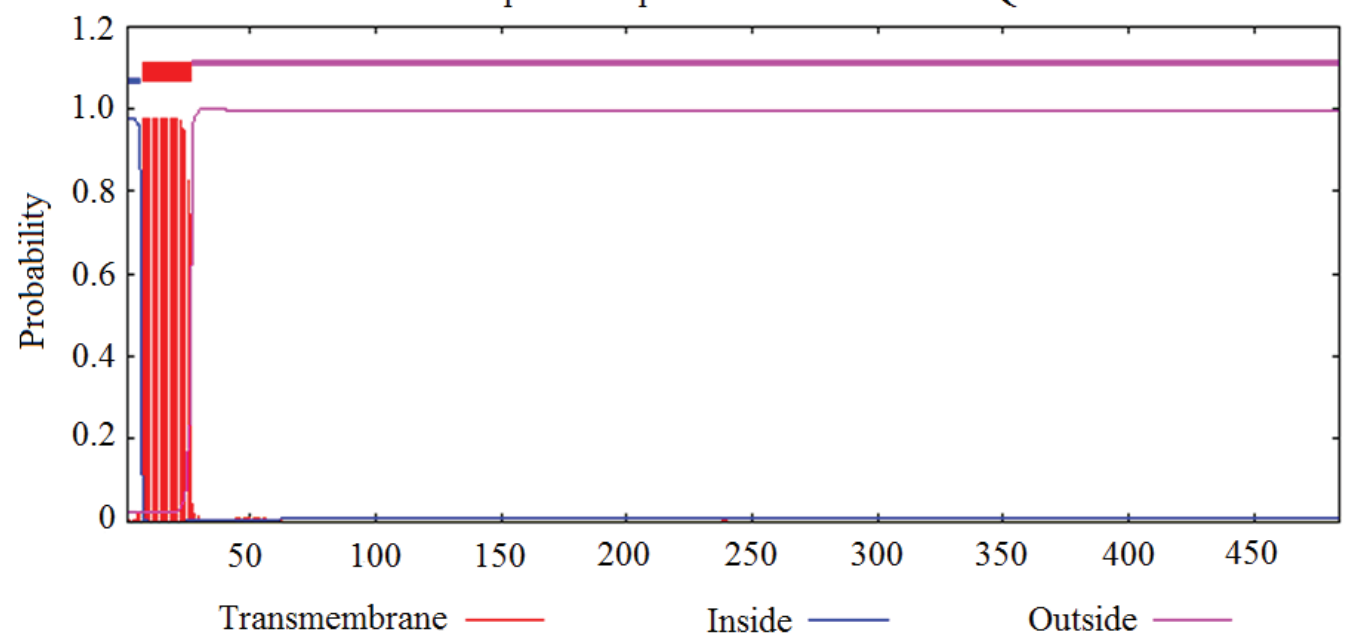

Figure 3. Transmembrane structure prediction of Ag5 using TMHMM software.

A

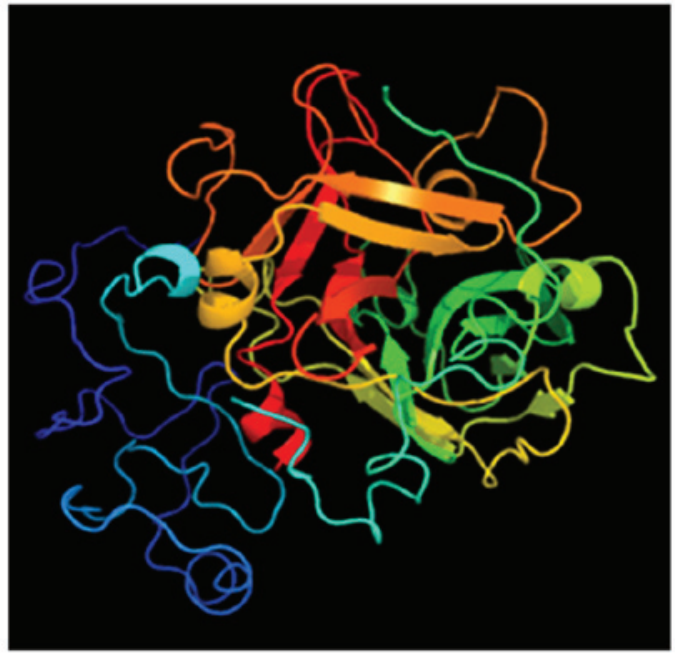

B

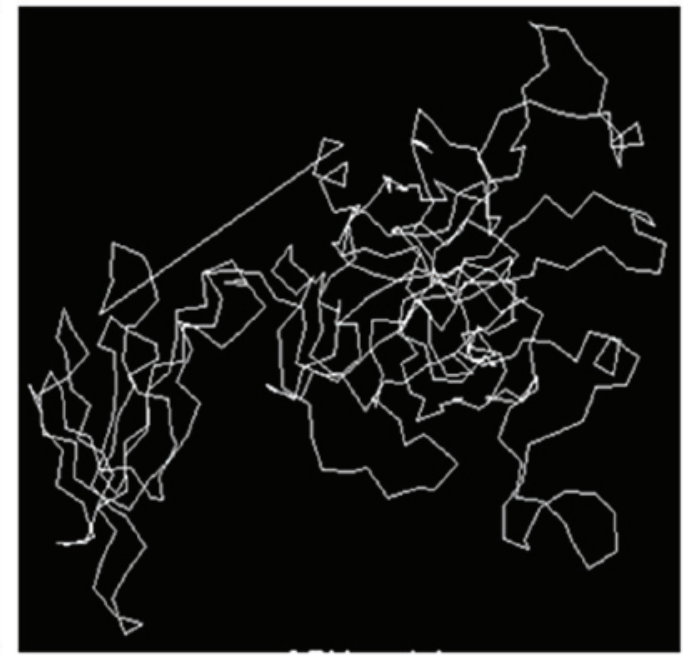

Figure 4. Tertiary structure prediction of Ag5: (A) 3DLigandSite; (B) CBS Prediction Servers. CBS, Center for Biological Sequence Analysis.

dll/EpitopePrediction.htm) (27). The amino acid sequence of Ag5 protein was input, the HLA molecule was set as HLA-A*0201, and the nonamers were set to 9 . The remaining parameters were not altered.

Prediction of various physicochemical properties of the Ag5 protein. Various physicochemical properties of the Ag5 protein were predicted by the online prediction software ProtParam (http://web.expasy.org/protparam/) (28). The sequence of Ag5 protein was input and five properties, including molecular weight, theoretical isoelectric point (pI), the instability index, the aliphatic index and the grand average of hydropathicity (GRAVY) were analyzed.

\section{Results}

Amino acid sequence encoded by the Ag5 gene. The open reading frame of $\mathrm{Ag} 5$ is $1,455 \mathrm{bp}$ in length and encodes 484 amino acids, as predicted by DNAman software (Fig. 1).
Secondary structure of the Ag5 protein. To analyze the immunogenicity of the $\mathrm{Ag} 5$ protein, its secondary structure was predicted using SOPMA Server, and the results revealed that $\alpha$-helixes, $\beta$-turns, random coils and extended strands accounted for 23.35, $10.95,41.32$, and $24.38 \%$ of the secondary structure, respectively. The predicted secondary structure results for the Ag5 protein are displayed in Fig. 2. The high proportion of random coils and extended strands in the structure of Ag5 protein suggest that the protein is likely to form antigenic epitopes.

The transmembrane structure of Ag5 protein was predicted using the online CBS prediction software TMHMM Server version 2.0. The inside, transmembrane and outside regions of Ag5 were located at positions 1-6, 7-26 and 27-484, respectively. The results are displayed in Fig. 3.

Tertiary structure of the Ag5 protein. The tertiary structure of the Ag5 protein was predicted using the online prediction server 3DLigandSite and compared with the structure from the CBS Prediction Servers. The results are displayed in Fig. 4. 

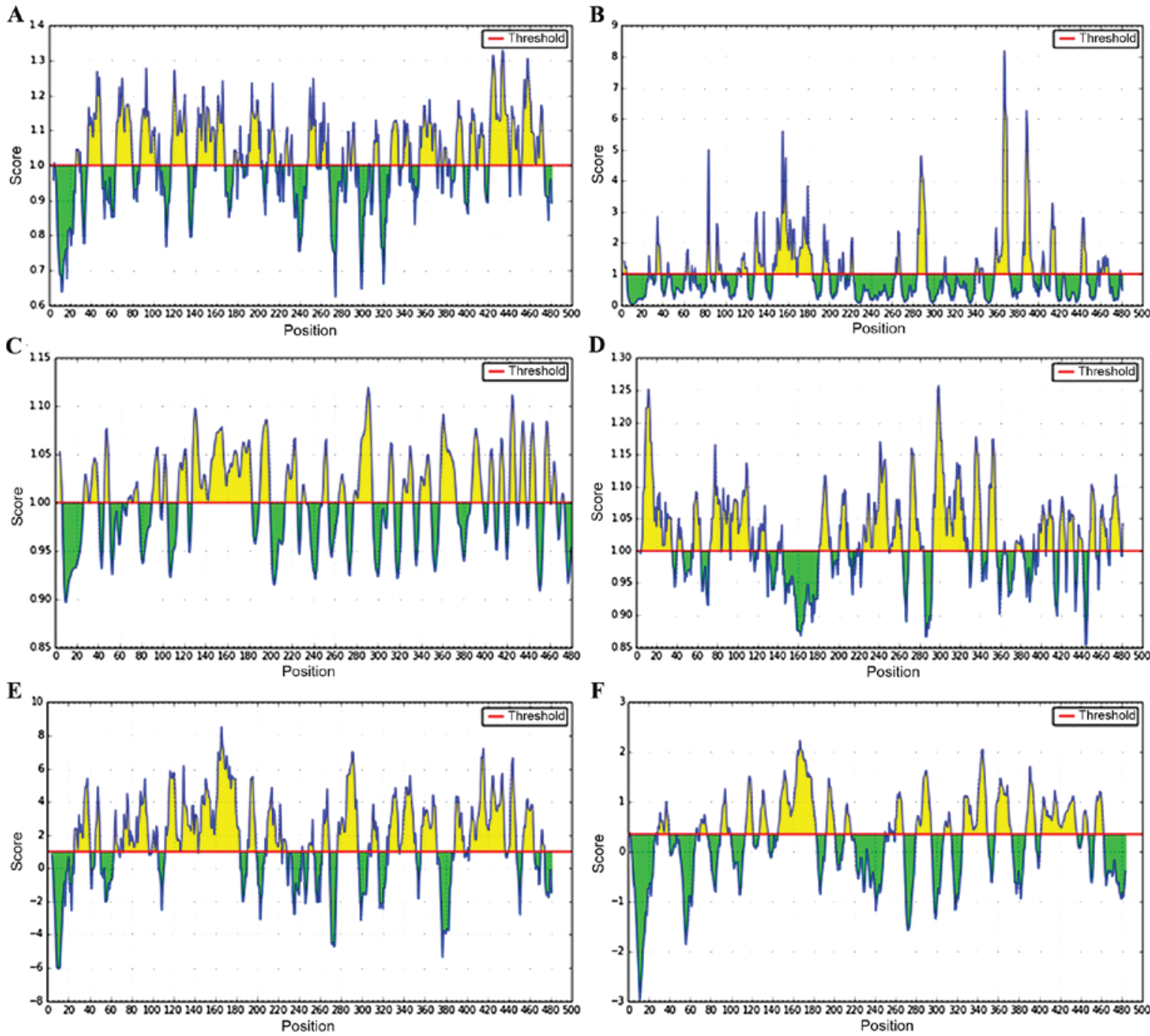

Figure 5. B cell epitope prediction for Ag5 using IEDB software: (A) Chou and Fasman $\beta$-turn; (B) Emini surface accessibility; (C) Karplus and Schulz flexibility; (D) Kolaskar and Tongaonkar antigenicity; (E) Parker hydrophilicity; and (F) BepiPredlinear epitope.

\section{Epitopes of the Ag5 protein}

$B$ cell epitopes of the Ag5 protein. B cell epitopes of Ag5 protein were predicted using the online prediction software IEDB (Fig. 5). High-scoring regions of $\beta$-turn were located at positions $26-30,36-50,64-80,87-102,117-131,142-168$, 177-202, 210-216, 248-267, 327-346, 355-396, 403-416, and 422-473, and the accessible surface areas were located at positions 91-96, 116-121, 128-138, 146-183, 195-200, 284-293, 340-347, 359-373, 385-395, 412-417, and 460-468. The flexible regions were $27-40,46-50,68-77,90-104,114-123,127-182$, 190-199, 214-225, 249-253, 264-269, 278-295, 310-314, 323-333, 339-349, 358-373, 387-397, 403-408, 413-417, $423-428,423-428,432-447$, and $455-473$, and the possible antigenic regions proved to be 5-35, 57-63, 73-83, 85-92, 94-112, 121-128, 181-192, 201-209, 224-249, 252-262, 270-284, 293-325, 333-341, 348-355, 375-380, 398-404, 406-412, $418-424,427-432,448-454,459-465$, and 469-479. Areas that had high hydrophilicity were located at positions $26-40,45-50$, 64-67, 70-96, 100-105, 113-183, 191-198, 210-228, 249-253, 263-268, 277-296, 310-315, 326-351, 357-371, 389-397, 403-436, 440-447, 454-464, and 471-474, and the linear epitopes were demonstrated to be 29-39, 70-76, 90-99, 114-121,
128-136, 146-182, 193-201, 211-216, 260-267, 280-294, 308-314, 326-349, 357-372, 387-396, 402-436, 443-447, and 454-462.

A combination of the results predicted by the different parameters indicated that the B cell epitopes were located at positions 27-39, 70-80, 117-130, 146-168, 250-262, 284-293, 339-349, 359-371, 403-412, and 454-462.

T cell epitopes of the Ag5 protein. To increase the accuracy of the prediction, $\mathrm{T}$ cell epitopes were predicted using the online prediction software BIMAS together with SYFPEITHI. There were 20 high-scoring regions, and the results are presented in Table I. These two software programs use different predicting systems. The BIMAS software resulted in high scores ranging from 3.932 to 1,737.776; however, the high scores predicted by the SYFPEITHI software ranged between 19 and 27. These high-scoring regions had strong potential of forming epitopes regardless of the difference.

Comparing the results predicted by the two online programs, seven high-scoring T cell epitopes were found to be located at positions 52-60, 57-65, 182-190, 231-239, 273-281, $318-326$ and 467-475. 
Table I. Major histocompatibility complex-Inonamer T cell epitope using BIMAS and SYFPEITHI.

\begin{tabular}{|c|c|c|c|c|c|c|}
\hline \multirow[b]{2}{*}{ No. } & \multicolumn{3}{|c|}{ BIMAS software } & \multicolumn{3}{|c|}{ SYFPEITHI software } \\
\hline & $\begin{array}{l}\text { Initiation } \\
\text { site }\end{array}$ & $\begin{array}{l}\text { Amino acid } \\
\text { sequence }\end{array}$ & $\begin{array}{l}\text { Score } \\
\text { site }\end{array}$ & $\begin{array}{l}\text { Initiation } \\
\text { sequence }\end{array}$ & Amino acid & Score \\
\hline 1 & 52 & LMFNRSLFV & 1737.776 & 395 & SLNEIRVSI & 27 \\
\hline 2 & 467 & WLYNSVGSV & 306.118 & 467 & WLYNSVGSV & 27 \\
\hline 3 & 318 & ALLRLETPV & 257.342 & 318 & ALLRLETPV & 25 \\
\hline 4 & 476 & IQWINRYAV & 99.501 & 18 & AALGLELTL & 24 \\
\hline 5 & 231 & TLITPRWVL & 65.689 & 273 & LLVRAGDTV & 24 \\
\hline 6 & 273 & LLVRAGDTV & 57.937 & 231 & TLITPRWVL & 23 \\
\hline 7 & 9 & IVFVCLFAT & 54.713 & 16 & ATAALGLEL & 22 \\
\hline 8 & 395 & SLNEIRVSI & 42.774 & 52 & LMFNRSLFV & 22 \\
\hline 9 & 237 & WVLTAAHCL & 31.814 & 57 & SLFVYRENI & 22 \\
\hline 10 & 299 & LVVIHPDWV & 27.882 & 182 & RLPYSLKIL & 22 \\
\hline 11 & 182 & RLPYSLKIL & 20.145 & 213 & NASEGLTRL & 22 \\
\hline 12 & 57 & SLFVYRENI & 18.915 & 50 & ATLMFNRSL & 21 \\
\hline 13 & 8 & WIVFVCLFA & 17.282 & 226 & IICGGTLIT & 21 \\
\hline 14 & 272 & FLLVRAGDT & 16.488 & 6 & PLWIVFVCL & 20 \\
\hline 15 & 442 & NEEDGRWYV & 14.638 & 24 & LTLDPDELV & 20 \\
\hline 16 & 24 & LTLDPDELV & 12.207 & 225 & NIICGGTLI & 20 \\
\hline 17 & 471 & SVGSVIQWI & 11.548 & 247 & PIFGSSNAL & 20 \\
\hline 18 & 11 & FVCLFATAA & 7.599 & 327 & NIESDGAGV & 20 \\
\hline 19 & 6 & PLWIVFVCL & 7.411 & 330 & SDGAGVACV & 20 \\
\hline 20 & 230 & GTLITPRWV & 3.932 & 189 & ILGGKSAKS & 19 \\
\hline
\end{tabular}

BIMAS, BioInformatics and Molecular Analysis Section.

Physicochemical properties of the Ag5 protein. The molecular weight of $\mathrm{Ag} 5$ is $54,874.8 \mathrm{Da}$, and its theoretical $\mathrm{pI}$ is 6.36 . The instability index is computed to be 55.78 , classifying the protein as unstable. The aliphatic index and GRAVY were found to be 69.32 and -0.525 , respectively.

\section{Discussion}

In China, cystic echinococcosis is a public health problem requiring a prompt solution. Furthermore, the infection region is gradually expanding (29). Currently, surgery in combination with chemotherapy is the first choice as a treatment strategy for cystic echinococcosis; however, it is not completely effective and the disease recurs. Immune prevention may be an effective measure to prevent the epidemic of cystic echinococcosis. Thus, developing a novel and effective vaccine is considered to be important.

It has been confirmed that $\operatorname{Ag} 5$ is expressed in all stages of the life cycle of $E$. granulosus (18), such as the tegument of the protoscolex, the embryonic membrane of eggs, the surface of oncospheres and adults. Thus, Ag5 presents as a promising vaccine for cystic echinococcosis.

Obtaining information regarding antigen epitopes will facilitate the development of epitope vaccines. Previously, epitope prediction was performed using a single parameter and its accuracy was limited. Now, as a result of the development of bioinformatics, the prediction of epitopes is accurate and simple. Making predictions using a multi-parameter and multi-method analysis improves the accuracy of epitope prediction greatly. In a study by Li et al (30), HLA-A*0201, HLA-A*1101 and HLA-A*2401 cytotoxic T lymphocyte restricted epitopes of platelet membrane glycoprotein IIb/IIIa (GP IIb/IIIa) antibody of human and mice were predicted using SYFPEITHI, RANKPEP, BIMAS, SVMHC, PREDEP, MHCPRED and PROPRED software, and the T cell epitopes of GP IIb/IIIa antibody were predicted. In a different study conducted by Shen et al (31), the secondary structure and surface characteristics of the follicle stimulating hormone receptor (FSHR) extracellular domain were analyzed using DNAStar Protean software, and B cell epitope prediction was conducted using alternative online software. The possible antigenic epitopes of the FSHR extracellular domain were predicted, the peptides of the epitopes were synthesized and the immunogenicity of these peptides was determined.

In the current study, the transmembrane structure of $\mathrm{Ag} 5$ was predicted and inside, transmembrane and outside regions were identified. The transmembrane region was stable and it could not alter or form epitopes easily. The outside region, which constituted the majority of the structure, was located at position 27-484. The flexible areas usually appeared here and this region could potentially form epitopes.

The secondary structure of the $\mathrm{Ag} 5$ protein was predicted, as it was closely associated with antigenic features. The $\alpha$-helixes and extended strands are regular structures in the secondary structure of the Ag5 protein. They are not deformed readily, as hydrogen bonds maintain the stability of their 
structure. However, ligand binding is difficult as the $\alpha$-helixes and extended strands are located inside the protein. By contrast, random coils and $\beta$-turns are located at the surface of Ag5, and they are suitable for ligand binding. Therefore, it is possible for these to form epitopes. As predicted by the SOPMA Server software, the proportion of $\alpha$-helixes was $23.35 \%$ and that of the extended strands was $24.38 \%$. Thus, it was inferred from the results that the Ag5 protein has good stability. Furthermore, random coils and $\beta$-turns, which represent potential epitope regions, account for 41.32 and $10.95 \%$ of the protein, respectively.

The tertiary structure is based on the secondary structure of the protein, and it is a three-dimensional globular structure composed of further coiling and folding of secondary structure elements, such as $\alpha$-helixes, $\beta$-turns, random coils and extended strands. The tertiary structure clarified that the random coils and $\beta$-turns are located at the surface of Ag5, and are suitable for ligand binding. These regions form epitopes easily. Therefore, the tertiary structure prediction was a necessary supplement to the prediction of Ag5 antigenic epitopes.

To improve the accuracy of the $\mathrm{B}$ cell epitope prediction, a multi-parameter analysis was utilized. The $\beta$-turn parameter prediction indicated that the $\beta$-turn is one of the normal types in the secondary structure of the protein. Hydrogen bonds alter the direction of the peptide chains, similarly to a U-model structure. Epitopes are able to easily form in these regions. Accessibility reflects the possibility of being found on the surface; the higher the accessibility, the more likely epitopes are to form. The flexibility parameter prediction demonstrates the ability of the protein to bend and fold. With a greater flexibility, the polypeptide skeleton of a protein has a strong capacity to bend and fold, thus promoting the formation of a secondary structure. The antigenic propensity demonstrated the immunogenic regions of the Ag5 protein. Those regions with high antigenic propensity are closely associated with the epitopes of the protein. The hydrophilicity parameter prediction explains the position of hydrophilic residues in the amino acid sequence of the protein. The hydrophilic residues are located on the surface of the protein and are suitable for ligand binding. The dominant epitopes are more likely to be located in regions with a high hydrophilicity. In combination with these parameters, the $\mathrm{B}$ cell epitopes of the Ag5 protein were predicted using IEDB software. Potential epitopes were revealed and were located at positions 27-39, 70-80, 117-130, 146-168, 250-262, 284-293, 339-349, 359-371, 403-412 and 454-462.

In the Chinese population, HLA-A*0201 is the most common HLA-I molecule (32). The accuracy of the MHC-I epitope prediction in the prediction of the $\mathrm{T}$ cell epitopes is declared to be $\leq 90 \%$. To increase the accuracy of the prediction, the T cell epitopes were predicted using BIMAS and SYFPEITHI software. Taken together, the $\mathrm{T}$ cell epitopes of the Ag5 protein were found to be located at positions 52-60, 57-65, 182-190, 231-239, 273-281, 318-326, and 467-475.

The aim of the present study was to obtain the bioinformatic characteristics of the Ag5 protein and analyze its immunogenicity. The results of the secondary structure prediction demonstrated that there are potential epitopes in the protein. The B cell epitopes were found to be located at positions 27-39, 70-80, 117-130, 146-168, 250-262, 284-293, 339-349, 359-371, 403-412, and 454-462; whereas the T cell epitopes were found to be located at positions 52-60, 57-65, 182-190, 231-239, 273-281, 318-326, and 467-475. These regions possess great potential for forming epitopes.

In conclusion, the current study predicts significant biological data for E. granulosus Ag5 to provide a theoretical basis for investigating its antigenicity and provides a theoretical foundation for epitope vaccine development for cystic echinococcosis.

\section{Acknowledgements}

Project support was provided, in part, by the training Programs of Innovation and Entrepreneurship for College Students in Jiangsu Province (grant no. 201510313017Z), the National Natural Science Foundation of China (grant no. 81501762), the Talents Scientific Research Foundation of Xuzhou Medical University (grant no. D2015004), the Natural Science Foundation of the Jiangsu Higher Education Institutions (grant no. 15KJB310025) and the Jiangsu Planned Projects for Postdoctoral Research Funds (grant no. 1501061A).

\section{References}

1. Wang $\mathrm{K}$, Zhang $\mathrm{X}$, Jin Z, Ma H, Teng Z and Wang L: Modeling and analysis of the transmission of Echinococcosis with application to Xinjiang Uygur Autonomous Region of China. J Theor Biol 333: 78-90, 2013.

2. Torgerson PR: The emergence of echinococcosis in central Asia. Parasitology 140: 1667-1673, 2013.

3. Moldovan R, Neghina AM, Calma CL, Marincu I and Neghina R: Human cystic echinococcosis in two south-western and central-western Romanian counties: A 7-year epidemiological and clinical overview. Acta Trop 121: 26-29, 2012.

4. Siracusano A, Delunardo F, Teggi A and Ortona E: Host-parasite relationship in cystic echinococcosis: An evolving story. Clin Dev Immunol 2012: 639362, 2012.

5. Liu CY, Ma XM, Ding JB and Shen HM: Different host status of echinococcus infection in China. Chin J Zoonoses 25: 586-588, 2009.

6. Nasrieh MA, Abdel-Hafez SK, Kamhawi SA, Craig PS and Schantz PM: Cystic echinococcosis in Jordan: Socioeconomic evaluation and risk factors. Parasitol Res 90: 456-466, 2003.

7. Gonlugur U, Ozcelik S, Gonlugur TE and Celiksoz A: The role of Casoni's skin test and indirect haemagglutination test in the diagnosis of hydatid disease. Parasitol Res 97: 395-398, 2005

8. Kalantari E, Bandehpour M, Pazoki R, Taghipoor-Lailabadi N, Khazan H, Mosaffa N, Nazaripouya MR and Kazemi B: Application of recombinant Echinococcus granulosus antigen B to ELISA kits for diagnosing hydatidosis. Parasitol Res 106: 847-851, 2010.

9. Tawfeek GM, Elwakil HS, El-Hoseiny L, Thabet HS, Sarhan RM, Awad NS and Anwar WA: Comparative analysis of the diagnostic performance of crude sheep hydatid cyst fluid, purified antigen $B$ and its subunit (12 Kda), assessed by ELISA, in the diagnosis of human cystic echinococcosis. Parasitol Res 108: 371-376, 2011.

10. Wen H, Aji T and Shao YM: Diagnosis and management against the complications of human cystic echinococcosis. Front Med China 4: 394-398, 2010.

11. Lightowlers MW, Lawrence SB, Gauci CG, Young J, Ralston MJ, Maas D and Heath DD: Vaccination against hydatidosis using a defined recombinant antigen. Parasite Immunol 18: 457-462, 1996.

12. Strohmaier K, Franze R and Adam KH: Location and characterization of the antigenic portion of the FMDV immunizing protein. J Gen Virol 59: 295-306, 1982.

13. Kouguchi H, Matsumoto J, Katoh Y, Oku Y, Suzuki T and Yagi K: The vaccination potential of EMY162 antigen against Echinococcus multilocularis infection. Biochem Biophys Res Commun 363: 915-920, 2007.

14. Kouguchi H, Matsumoto J, Yamano K, Katoh Y, Oku Y, Suzuki T and Yagi K: Echinococcus multilocularis: Purification and characterization of glycoprotein antigens with serodiagnostic potential for canine infection. Exp Parasitol 128: 50-56, 2011 
15. Katoh Y, Kouguchi H, Matsumoto J, Goto A, Suzuki T, Oku Y and Yagi K: Characterization of emY162 encoding an immunogenic protein cloned from an adult worm-specific cDNA library of Echinococcus multilocularis. Biochim Biophys Acta 1780: 1-6, 2008.

16. Lorenzo C, Salinas G, Brugnini A, Wernstedt C, Hellman U and González-Sapienza G: Echinococcus granulosus antigen 5 is closely related to proteases of the trypsin family. Biochem J 369: 191-198, 2003.

17. Lightowlers MW, Liu DY, Haralambous A and Rickard MD: Subunit composition and specificity of the major cyst fluid antigens of Echinococcus granulosus. Mol Biochem Parasitol 37: 171-182, 1989.

18. Li Y, Xu H, Chen J, Gan W, Wu W, Wu W and Hu X: Gene cloning, expression, and localization of antigen 5 in the life cycle of Echinococcus granulosus. Parasitol Res 110: 2315-2323, 2012.

19. Paul M and Stefaniak J: Detection of specific Echinococcus granulosus antigen 5 in liver cyst bioptate from human patients. Acta Trop 64: 65-77, 1997.

20. Yarzabal LA, Bout DT, Naquira FR and Capron AR: Further observations on the specificity of antigen 5 of Echinococcus granulosus. J Parasitol 63: 495-499, 1977

21. Geourjon C and Deléage G: SOPMA: Significant improvements in protein secondary structure prediction by consensus prediction from multiple alignments. Comput Appl Biosci 11: 681-684, 1995.

22. Melén K, Krogh A and von Heijne G: Reliability measures for membrane protein topology prediction algorithms. J Mol Biol 327: 735-744, 2003.

23. Wass MN, Kelley LA and Sternberg MJ: 3DLigandSite: Predicting ligand-binding sites using similar structures. Nucleic Acids Res 38: W469-W473, 2010.

24. Nielsen $M$, Lundegaard $C$, Lund $O$ and Petersen $T N$ : CPHmodels-3.0-remote homology modeling using structure-guided sequence profiles. Nucleic Acids Res 38: W576-W581, 2010.
25. Vita R, Zarebski L, Greenbaum JA, Emami H, Hoof I, Salimi N, Damle R, Sette A and Peters B: The immune epitope database 2.0. Nucleic Acids Res 38 (Database): D854-D862, 2010.

26. Parker KC, Bednarek MA and Coligan JE: Scheme for ranking potential HLA-A2 binding peptides based on independent binding of individual peptide side-chains. J Immunol 152: $163-175,1994$.

27. Rammensee H, Bachmann J, Emmerich NP, Bachor OA and Stevanović S: SYFPEITHI: Database for MHC ligands and peptide motifs. Immunogenetics 50: 213-219, 1999.

28. Gasteiger E, Gattiker A, Hoogland C, Ivanyi I, Appel RD, and Bairoch A: ExPASy: The proteomics server for in-depth protein knowledge and analysisNucleic Acids Res 31: 3784-3788, 2003.

29. Li YJ, Wang J, Zhao H, Jia HY, Li B, Ma XM, Wen H and Ding JB: Bioinformatics prediction on Eg95 antigen epitopes of Echinococcus granulosus. Chin J Zoonoses 27: 892-895, 2011.

30. Li Z, Zhang M, Hu H, Liu S and Lu Z: On predicting the T cell and B cell epitopes of platelet membrane glycoprotein II b/III a antibody from human and mice]. Sheng Wu Yi Xue Gong Cheng Xue Za Zhi 27: 1146-1151, 2010 (In Chinese).

31. Shen ZG, Yan P, He W, Chen Z, He H, Zhang J, Yang X, Wu Y, Liang $\mathrm{Z}$ and Li J: Prediction of the secondary structure and the B cell epitope of the extracellular domin of FSHR. J Chongqing Med Univ 35: 1317-1320, 2010

32. Yan C, Wang R, Li J, Deng Y, Wu D, Zhang H, Zhang H, Wang L, Zhang C, Sun H, et al: HLA-A gene polymorphism defined by high-resolution sequence-based typing in 161 Northern Chinese Han people. Genomics Proteomics Bioinformatics 1: 304-309, 2003. 